\title{
The NORT: Network of Oriental Robotic Telescopes
}

\author{
Roger Hajjar \\ Department of Sciences, Notre-Dame University, Po-Box 72 Zouk \\ Mikael, Lebanon (e-mail: rhajjar@ndu.edu.lb)
}

François R. Querci \& Monique Querci

Observatoire Midi-Pyrénées, 14 Av. Edouard Belin, 31400 Toulouse, France (e-mail: querci@ast.obs-mip.fr)

\begin{abstract}
In this paper, we present the NORT, a network of oriental robotic telescopes originally proposed in 1993 by Querci \& Querci to study stellar variability. The NORT project covers all of northern Africa and Asia near the tropic of Cancer. The interest generated among a number of astronomers in countries from the Arab World and Asia offers a solid basis for the rebirth of astronomy and astrophysics and space sciences in developing countries. The number of national projects for telescopes in different countries makes the NORT a framework of choice to coordinate and stimulate these separate efforts by the creation of a NORT steering committee.
\end{abstract}

\section{Introduction}

The NORT was initially proposed in 1993 by Querci et al. as a complement to the GNAT. The aim was to have a dedicated network of robotic telescopes completely devoted to the study of long-term variability of stellar objects. They planned initially to use stations of three 1-m telescopes with photometers. The development of large CCDs lead to the idea of a network of small $(0.6-1 \mathrm{~m})$ to medium $(1 \mathrm{~m}-2 \mathrm{~m})$ class telescopes.

The idea of establishing stations at or near the tropic of Cancer comes from the interesting astronomical possibilities, that will be discussed later, presented by this region of the world. Thus locations from North Africa to Asia where sought for the establishment of the Network as a complement to GNAT, and a French-Arab project was initiated. It is interesting to note that this longitude range covers mostly developing countries (DCs). In this paper we seek to develop interest in a network and the NORT in particular which will lead to the development of astronomy in these countries. The current status of the project is shown as well as its future prospects. 


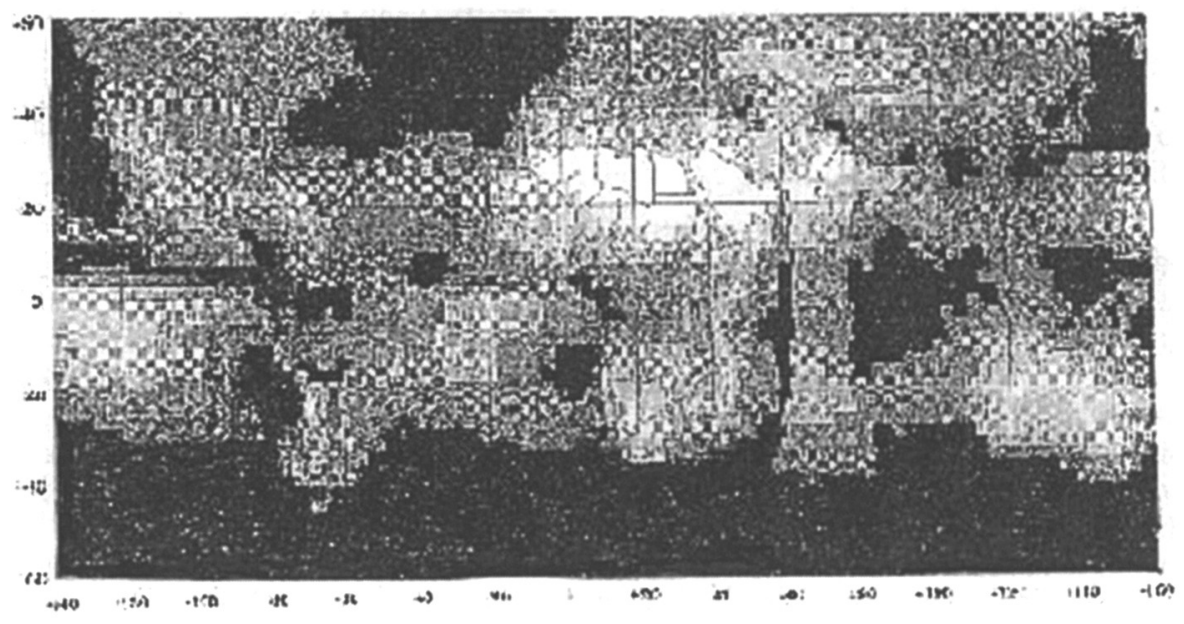

Figure 1. Mean annual nebulosity obtained from meteorological satellite data. White represents nebulosity $>30 \%$, Black $<70 \%$. Map produced with a $250 \mathrm{~km}$ square mesh.

\section{The NORT}

The NORT proposes to link a number of stations placed in excellent astronomical sites to monitor photometrically, spectroscopically and polarimetrically variable objects. The NORT would like to achieve 24-hour, year round monitoring of selected targets. Robotic telescopes are an excellent choice for that task. By robotic, we mean a fully automated observatory where all functions are operator independent, a system that might basically need a kick once every few weeks to run.

\subsection{Locations}

To determine the best possible sites, meteorological data have been used to build a map of mean nebulosity for the whole world as shown in Querci \& Querci (1998). The map is reproduced from their article in Figure 1. White area represents very low nebulosity. The mesh used to average data is $250 \times 250 \mathrm{~km}$. The data then should be taken as indicative of possible excellent astronomical sites. It is noticeable that both North Africa and the Middle East show compelling evidence for exceptional conditions, that is dry, semi-arid and with high peaks (the Atlas mountains in North Africa, the peaks south of Jordan, Lebanon and the Anti-Lebanon mountain ranges, Mount Sinai, the South-Arabia mountain chain, etc...).

The presence of DCs in this range makes it interesting because the current state of development makes preservation of good astronomical conditions at these sites quite possible. Based on Figure 1 and on geographical information one can determine possible locations for observatories, as shown in Figure 2 


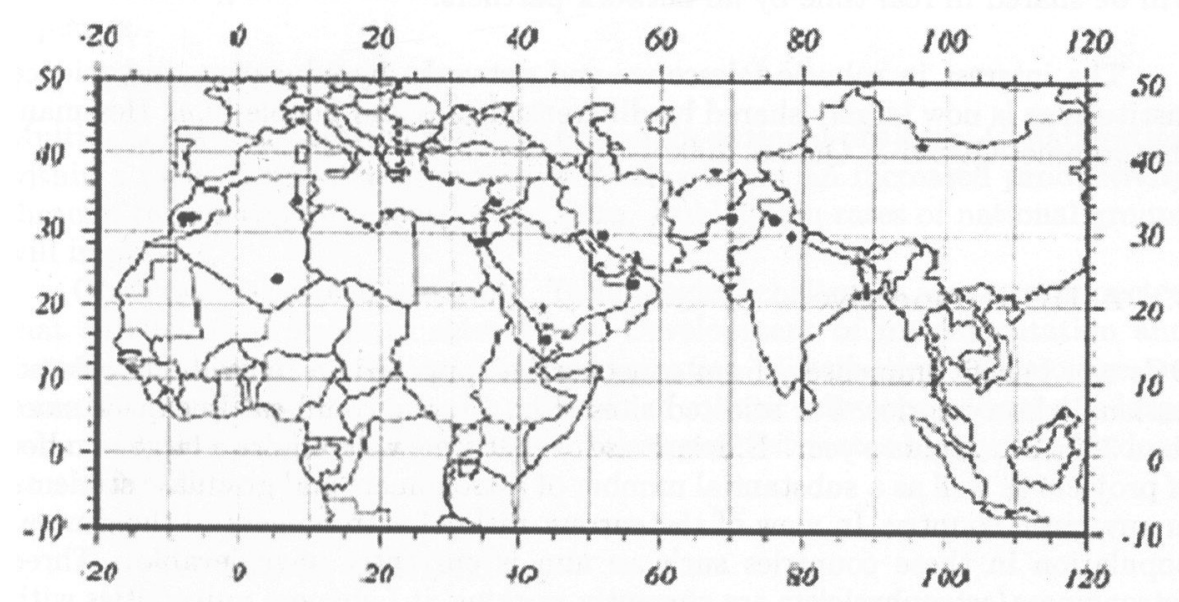

Figure 2. Sites chosen based on the meteorological map shown in Fig.1 and geographical information (presence of peaks). Requires in situ site testing to confirm.

(Querci \& Querci, 1998). Filled circles represent exceptional sites, stars represent good astronomical locations. Future steps will involve studying satellite data with a much smaller mesh $(3-5 \mathrm{~km})$ and in three wavebands, as well as the implementation of preservation measures for selected sites (Querci \& Querci, 1999). Some countries have already started or are planning such studies. Tunisia has already completed the site selection process (Jaidane, 2000), as well as Syria (Al-Mousli, 2000). Libya is planning such a study.

\subsection{Objectives}

Although all countries in this longitude range are considered to be DCs, almost all have had a long tradition in (ancient) astronomy. Since it provides grounds for a rebirth and/or development of astronomy, NORT would like then to contribute to astronomy education as well as research development in DCs.

Its aims are:

- Building capacity in basic space sciences: education in universities, technical staff training, and building up research potential.

- To help establish national observatories within the framework of NORT.

- A permanent network for the study of long-term stellar variability by spectroscopy, photometry, and polarimetry, and exo-planet research as chief objectives to increase collaboration among partner countries.

- Providing ground for collaboration with and observations at larger facilities (NGST, VLTI, Keck ...) based on NORT observations.

- To produce a large database of variable-star observations for studies like astroseismology and circumstellar environments. All data collected by the NORT 
will be shared in real time by all network partners.

The interest in robotic telescopes and networks by education and science institutions is now largely shared by different groups and people (e.g., Hessman, this volume; Querci \& Querci, 2000).

\section{A DC Perspective}

DCs can benefit immensely from a network as opposed to individual, isolated national observatories. For selected sites (Fig. 2), one could easily expect more than 300 clear nights a year. Efficient use of that time will require a large number of projects as well as a substantial number of astronomers and graduate students in any given country. In view of the current ratio of astronomers to the general population in these countries such an aim is currently unachievable. Three astronomers/astrophysicists are currently working at Lebanese universities with a population of little less than 4 million (2000 estimate). Three of our students are currently pursuing graduate studies in France.

Furthermore, science budgets in DCs are very low compared to GDP. Allocation of funds is more competitive. Publication rates and their impact will thus play a major role. Arab League budgets in science amount to no more than USD 550 million for 23 countries. The Lebanese National Council for Scientific Research is granted an annual budget of USD 3-4 million, with an estimated GDP of USD 16 billion. An isolated observatory with a very small group would have difficulties using all the available clear nights and justifying expenses.

Isolation is also one of the main problems facing researchers in DCs. Lack of funds makes it difficult to travel extensively. Access to literature and important scientific information is also reduced because of its cost.

\section{NORT Benefits}

A network helps alleviate all or part of the above mentioned problems. Networked robotic telescopes with defined tasks will efficiently use all available clear nights. Furthermore, the focused research topic approach for a permanent network provides a reasonable framework for the development of astronomy at the national level, while benefitting from all the resources available due to other collaborators. The NORT would thus help at both the national level and the multinational level.

\subsection{National Observatories}

Small robotic telescopes $(0.6-1 \mathrm{~m})$ have many advantages:

- They are now widely available in standard configurations.

- Their low cost makes them accessible to national research budgets.

- Their manageable size does not require a large technical workforce which lowers their maintenance cost.

- They can be made available to advanced amateurs thus increasing their user base. 
NORT-organized summer schools will use local facilities to reduce training expenses.

\subsection{Networking}

Multinational collaboration will help to sustain national projects. Collaboration within a focused research approach will bring about an increased productivity through the sharing of resources and data. Publication rates of national groups will improve.

Observatories evolve and grow. Technological challenges are to be expected that benefit other fields of astronomy. Development of instrumentation and automatic systems, as well as data dissemination, distribution and storage are some of the main problems facing networks of robotic telescopes due to the large volume of data produced per night, per telescope. Estimates have yet to be done for the NORT.

\section{Current Status of NORT}

NORT has already generated interest in different groups and with individual researchers in different countries in this longitude range. Collaborators are from France, India, Jordan, Lebanon, Libya, Malaysia, Morocco, Pakistan, Syria, Tunisia, and Yemen. Iranians have also shown some interest in the project. More details can be found in Querci et al. (2000).

Since 1993, the initial proposal for NORT, a number of observatory projects have been in various stages of development. A non-exhaustive list includes (from Querci et al., 2000):

- 1.93 m Kottamya Observatory, Egypt, refurbished in 1996,

- Iraq has a German made $3.5-\mathrm{m}$ mirror that was going to be installed in a nearly complete building before the war,

- Jordan has a 40-cm operational observatory and a project for a $1.5-\mathrm{m}$ one,

- Kuwait is planning for a 1-m to 2-m class observatory to serve the Gulf area,

- Lebanese astronomers and scientists are proposing a 1-m class telescope,

- Libya is discussing the implementation of a 2-m class telescope,

- A 50-cm robotic telescope is being commissioned in Malaysia,

- Morocco has recently received a 55-cm Newton telescope from France that is being used for education,

- Pakistan has completed site selection studies for a 1.5-m project,

- Syria is planning for two university observatories or a 1-m class project,

- Tunisia has also completed site selection for 1-m class telescope,

- Yemen is considering a 1-m class national observatory.

NORT was approved by the sixth UN/ESA Workshop (Bonn, Germany, Sept. 1996), and subsequently endorsed by the United Nations General Assembly in December 1996, so it forms a follow-up project of the UN/ESA Workshops. Again, this decision was encouraged by the UN General Assembly on October 2000, following the recommendations issued by the ninth UN/ESA Workshop (Toulouse, France, June 2000). It is also considered by the Arab Union for Astronomy and Space Sciences (1998) as a key project for the development of Astronomy and Astrophysics in Arab countries. 


\section{Future Prospects}

We are currently in the process of establishing a steering and coordinating committee to synchronize efforts toward the actual establishment of some or all of the projects mentioned above. The committee will also implement plans to network already existing telescopes and to foster scientific collaboration between national groups. One of the main goals is to study the means for rapid and automated dissemination of data.

\section{Conclusions}

The NORT plans a permanent network to study stellar variability with stations located at/or near the tropic of Cancer. It will help to foster astronomy in its partner countries while contributing to the regional and international development of this science. In view of the number of observatory projects that have arisen, the NORT is now ready to move toward the creation of a steering committee to further national projects. One of the main challenges lying ahead will be to make sure that the observatories, while contributing to NORT, can still be used for training and educational purposes locally. This requires a hybrid mode of operation combining both manual and robotic techniques.

\section{References}

Al-Mousli, M. 2000, in Fourth Arab Conference for Astronomy and Space Sciences, Mafraq, Jordan, August 28-31, 2000

Hessman, F.V. 2001, this volume

Jaidane, N. 2000, in Ninth UN/ESA Workshop on Basic Space Science: Satellites and Networks of Telescopes, Toulouse, France, 27-30 June, 2000

Querci, F.R., Querci, M., Hajjar, R., Al-Naimy, H., Konsul, K. 2000, in Ninth UN/ESA Workshop on Basic Space Science: Satellites and Networks of Telescopes, Toulouse, France, 27-30 June, 2000

Querci, F.R., Querci, M., Kadiri, S., de Rancourt, L. 1993, in IAU Colloquium 136 on Stellar Photometry, eds. E. Elliott and C.J. Butler (Dublin Institute for Advanced Studies), 122

Querci, F.R., Querci, M. 2000, Astrophys. \& Space Sc., 273, 257

Querci, F.R., Querci, M. 1999, in IAU Symp. 196 on Preserving the Astronomical Sky: an IAU/COSPAR/UN Special Environment Symposium, eds. R.J. Cohen and W.T. Sullivan, 12-16 July 1999, Vienna, Austria, in press

Querci, F.R., Querci, M. 1998, in A.S.P. Conf. Series on Preserving the Astronomical Windows, eds. S. Isobe and T. Hirayama, 139, 135 